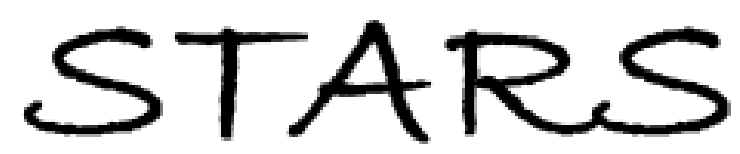

University of Central Florida

STARS

Faculty Bibliography 2000s

Faculty Bibliography

$1-1-2005$

\title{
Waveguide array-grating compressors
}

Sotiris Droulias

Kyriakos Hizanidis

Demetrios N. Christodoulides

University of Central Florida

Roberto Morandotti

Find similar works at: https://stars.library.ucf.edu/facultybib2000

University of Central Florida Libraries http://library.ucf.edu

This Article is brought to you for free and open access by the Faculty Bibliography at STARS. It has been accepted for inclusion in Faculty Bibliography 2000s by an authorized administrator of STARS. For more information, please contact STARS@ucf.edu.

\section{Recommended Citation}

Droulias, Sotiris; Hizanidis, Kyriakos; Christodoulides, Demetrios N.; and Morandotti, Roberto, "Waveguide array-grating compressors" (2005). Faculty Bibliography 2000s. 5148.

https://stars.library.ucf.edu/facultybib2000/5148

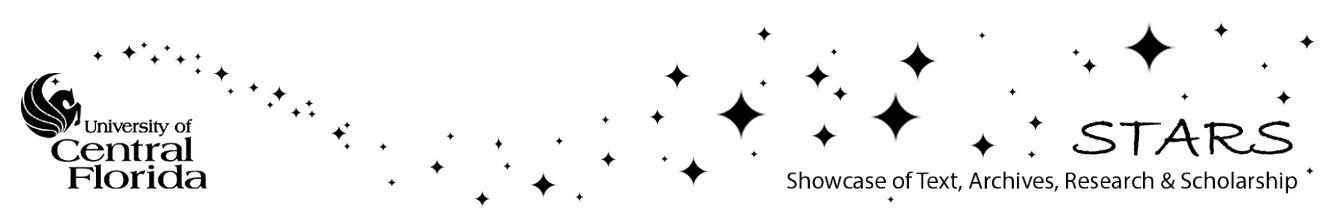




\title{
Waveguide array-grating compressors
}

\author{
Sotiris Droulias and Kyriakos Hizanidis ${ }^{a)}$ \\ School of Electrical and Computer Engineering, National Technical University of Athens, \\ 9 Iroon Polytechniou Str, Zografou, 15773, Athens, Greece
}

Demetrios N. Christodoulides

College of Optics and Photonics, CREOL, University of Central Florida, 4000 Central Florida Boulevard, Orlando Florida 32816

\author{
Roberto Morandotti \\ Institut National de la Recherche Scientifique, Université du Québec, 1650 Boul. Lionel Boulet, J3X 1S2, \\ Varennes, Canada
}

(Received 20 April 2005; accepted 29 July 2005; published online 21 September 2005)

\begin{abstract}
We show that efficient optical pulse compression can be achieved when normally dispersive nonlinear waveguide arrays are used in conjunction with dispersive elements such as gratings or other programmable phase filters. Our computations indicate that the compression resulting from such discrete arrays can be of better quality as compared to that obtained traditionally from a single nonlinear waveguide element. The performance of the array-grating compression method is assessed in normally dispersive highly nonlinear AlGaAs array systems. (C) 2005 American Institute of Physics. [DOI: 10.1063/1.2056578]
\end{abstract}

Optical pulse compression has always been an issue of great importance. ${ }^{1-7}$ These days femtosecond technology has advanced to the point where pulses below 6 fs can be routinely generated in the cavity of solid state lasers (such as Ti:sapphire) using Kerr lens modelocking and saturable absorber mirror techniques. ${ }^{8,9}$ Yet, inspite of these recent advances, there is constantly a need to develop alternative means for efficient pulse compression especially at the long wavelength range of $1.55 \mu \mathrm{m}$ which is of interest for telecommunications. ${ }^{6}$ In this spectral region pulse compression can be accomplished through several processes. These include, for example, soliton effects in anomalously dispersive and dispersion decreasing fibers, cross-phase modulation, and propagation in nonlinear fiber-loop mirrors, just to mention a few. ${ }^{6}$ One of the most efficient schemes through which optical pulse compression can be achieved is that involving a nonlinear normally dispersive optical fiber (or waveguide) followed by an anomalously dispersive element such as a pair of gratings or prisms. ${ }^{1-7}$ These compressors, better known as fiber-grating compressors, rely on the spectral broadening and linear chirp induced by the combined effects of self-phase modulation and normal dispersion in a waveguide. ${ }^{4}$ The purpose of the anomalously dispersive element that follows in these types of arrangements is to cancel the quadratic-like phase in the spectrum of the pulse or to reshuffle all the spectral components in the time domain. This in turn leads to pulse compression in time..$^{1-5}$

Very recently nonlinear wave propagation in discrete optical systems such as waveguide arrays has been the focus of considerable research. ${ }^{10,11}$ Such discrete structures are known to exhibit a wealth of properties resulting from their structural periodicity in the transverse direction. For instance, reverse or anomalous diffraction is possible when the waveguide lattice is operated near the edges of the spatial

${ }^{a}$ Author to whom correspondence should be addressed; electronic mail: kyriakos@central.ntua.gr
Brillouin zone, ${ }^{12}$ whereas discrete, gap, and vortex solitons can form under nonlinear conditions. ${ }^{10,13-15}$

Among the most important array structures used to experimentally explore these phenomena are those made from low-loss AlGaAs. AlGaAs, apart from having a high Kerr nonlinearity (is at least two orders of magnitude more nonlinear than silica glass) also happens to exhibit strong normal dispersion around $1.55 \mu \mathrm{m}$. In addition, at this wavelength region (corresponding to the AlGaAs midgap), two- and three-photon nonlinear absorption contributions are small and thus can be ignored. Quite recently the nonlinear dynamics of optical pulses have also been investigated in this material system. More specifically, Modotto et al. have studied the multi-peak spectra from 250-fs-long pulses (at $1520 \mathrm{~nm}$ ) in single AlGaAs waveguides ${ }^{16}$ and the possibility of discrete $\mathrm{X}$ waves in nonlinear normally dispersive waveguide arrays has also been suggested. ${ }^{17}$

In this letter we show that efficient optical pulse compression can be achieved when normally dispersive nonlinear waveguide arrays are used together with dispersive elements such as gratings or other programmable phase filters. Our simulations demonstrate that the compression resulting from such discrete arrays can be of better quality as compared to that obtained from a single nonlinear waveguide element. This is mainly due to the fact that in normally dispersive arrays, the optical pulse sheds its low intensity parts through discrete diffraction, and hence develops a rectangular-like profile of richer bandwidth. The performance of the arraygrating compression scheme is examined in AlGaAs array systems.

To study pulse compression in a waveguide array-grating configuration (as shown schematically in Fig. 1) let us consider a lattice of equally spaced, weakly coupled, singlemode waveguides. From coupled-mode theory, the modal field amplitude $U_{n}$ in the $n$th waveguide evolves according to ${ }^{10-17}$ 


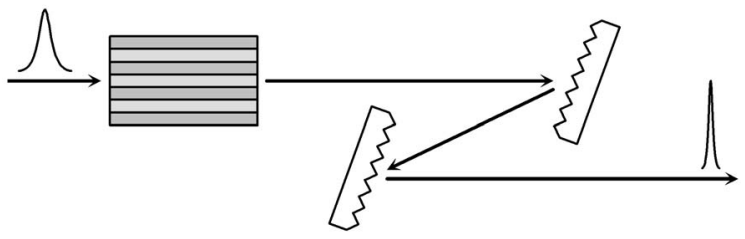

FIG. 1. A waveguide array-grating compressor arrangement.

$$
i \frac{\partial U_{n}}{\partial z}-\frac{\beta_{2}}{2} \frac{\partial^{2} U_{n}}{\partial t^{2}}+C\left[U_{n+1}+U_{n-1}\right]+k_{0} n_{2}\left|U_{n}\right|^{2} U_{n}=0,
$$

where $\beta_{2}$ represents the group velocity dispersion coefficient, $C$ is the linear coupling strength among waveguide sites, $k_{0}=2 \pi / \lambda_{0}$ and $n_{2}=\left(\hat{n}_{2} n\right) /\left(2 \eta_{0}\right)$. In the last expression, $\hat{n}_{2}=2.47 \times 10^{-13} \mathrm{~cm}^{2} / \mathrm{W}$ is the AlGaAs self-focusing Kerr coefficient, $n$ is the linear refractive index of AlGaAs and $\eta_{0}=\left(\mu_{0} / \varepsilon_{0}\right)^{1 / 2}$. The effective cross-sectional area of each waveguide element in the array is taken to be $5 \mu \mathrm{m}^{2}$. The array is considered to be $1 \mathrm{~cm}$ long and is assumed to involve 41 waveguides uniformly spaced at a distance of $10 \mu \mathrm{m}$. This array is used at $\lambda_{0}=1.55 \mu \mathrm{m}$ where the AlGaAs normal dispersion is $\beta_{2}=+1.3 \mathrm{ps}^{2} / \mathrm{m}$. The coupling constant is assumed to be $C=728 \mathrm{~m}^{-1}$ which is close to that encountered in previous experimental studies. ${ }^{12,13,15}$ In order to compare the performance of the array compressor against that obtained from a single waveguide element (from a standard fiber-grating arrangement), we solve the evolution equation $^{4}$

$$
i \frac{\partial U_{0}}{\partial z}-\frac{\beta_{2}}{2} \frac{\partial^{2} U_{0}}{\partial t^{2}}+k_{0} n_{2}\left|U_{0}\right|^{2} U_{0}=0 .
$$

This latter equation describes optical nonlinear propagation in a single AlGaAs waveguide element with the same physical properties assumed in the array. Equations (1) and (2) are solved numerically by means of a symmetrized split-step Fourier scheme the accuracy of which is checked against the conservation laws of these equations. In all our computations, we assume that the array is excited at the central waveguide (guide 21). In addition, both the array and the single waveguide are initially excited in time with a hyperbolic secant pulse of the same temporal width and peak power. In all the figures, $\left|U_{n}\right|^{2}=1$ represents the peak power initially used.

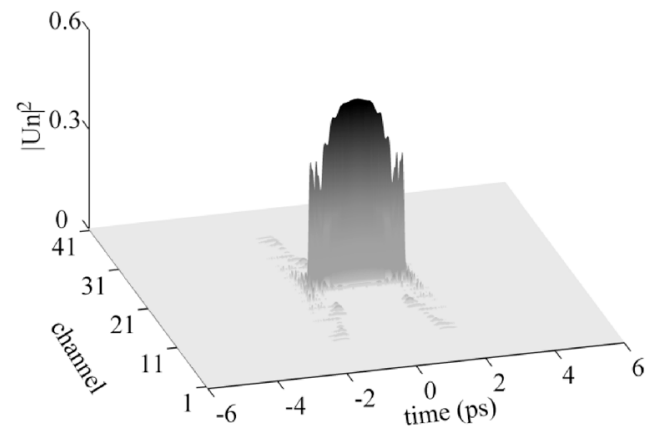

FIG. 2. Intensity profile at the output of a 1-cm-long AlGaAs array, when the central channel is excited with a sech-pulse of 1.76 ps FWHM duration and $1.5 \mathrm{~kW}$ peak power (three-dimensional depiction).
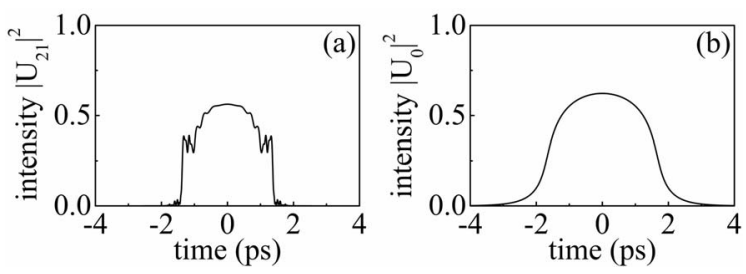

FIG. 3. Intensity profile at the output of (a) the central waveguide of an AlGaAs array; (b) a single AlGaAs waveguide. The parameters used are the same as in Fig. 2.

To analyze the proposed scheme we assume that a sech pulse of $1 \mathrm{ps}$ duration [1.76 ps intensity full width at half maximum (FWHM)] and $1.5 \mathrm{~kW}$ peak power excites the middle (guide 21) of the normally dispersive AlGaAs array. Figure 2 depicts the pulse intensity distribution (among waveguides) at the array output. The intensity pattern obtained is due to the interplay between discrete diffraction, dispersion, and nonlinearity. Because of self-phase modulation most of the power remains confined within the central waveguide, whereas the low-intensity components linearly disperse across the array through discrete diffraction, i.e., the pulse sheds its low intensity parts. Because of this process, the pulse in the central waveguide [shown in Fig. 3(a)] develops a rectangular-like intensity profile with extremely steep edges. Thin in turn further enriches its spectrum. For comparison, Fig. 3(b) illustrates the intensity expected from a single AlGaAs waveguide under the same conditions. Obviously there is a marked difference between these two configurations which is of course expected to impact their compression fidelity.

A comparison of the power spectra associated with these two systems is shown in Figs. 4(a) and 4(b). These results indicate that a pulse propagating in a nonlinear array develops a richer spectrum compared to that expected from a single waveguide element. In addition, the power spectrum resulting from the array is more uniform, i.e., less modulated. This strongly suggests that better compression ratios can be achieved from arrays. In the frequency domain, the spectrum of the pulse at the output of the central waveguide in the array can be expressed as $U(\omega)=|U(\omega)| \cdot \exp [i \cdot \phi(\omega)]$ where $|U(\omega)|$ is associated with the power spectrum. In general, the phase $\phi(\omega)$ can be expanded in a Taylor series as, that is $\phi(\omega)=\phi_{0}+\phi_{1}\left(\omega-\omega_{0}\right)+(1 / 2) \phi_{2}\left(\omega-\omega_{0}\right)^{2}+\ldots$, where the coefficients $\phi_{j}=d^{j} \phi(\omega) / d \omega^{j}$ are evaluated at the carrier frequency $\omega_{0}$. $\phi_{1}$ leads to a time shift, $\phi_{2}$ represents frequency chirp, and the remaining coefficients correspond to higher-order phase terms. Our computer simulations indicate that the spectrum phase $\phi(\omega)$ of the field at the output of the central array waveguide has a quadratic-like dependence on frequency. This is also the case when only a single AlGaAs
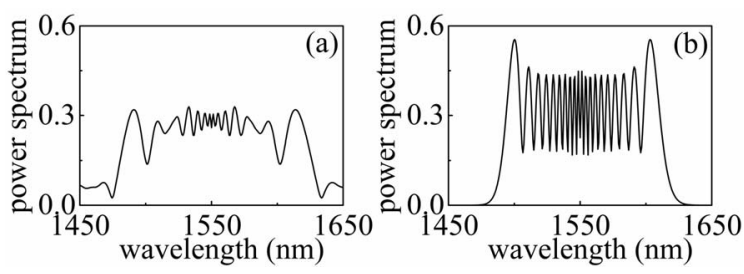

FIG. 4. Output power spectrum at $z=1 \mathrm{~cm}$ when an input pulse of $1.5 \mathrm{~kW}$ peak power and 1.76 ps FWHM is used; (a) from the central waveguide of an AlGaAs array; (b) from a single AlGaAs waveguide. 

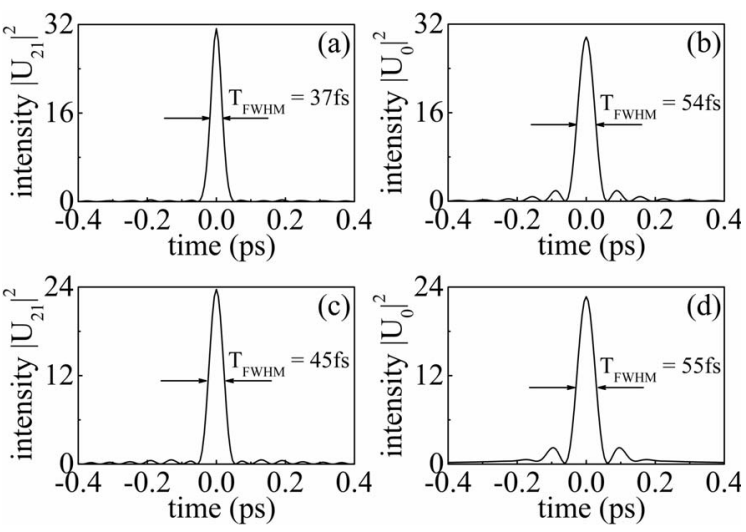

FIG. 5. Compressed pulse when the input is provided by (a),(c) the central waveguide of an AlGaAs array; (b),(d) an AlGaAs waveguide. (a),(b) ideal compression; (c),(d) compression with second-order correction only.

waveguide is used. In order to compress the pulse, an anomalously dispersive element is then used after the array. Best compression is expected when this dispersive structure totally cancels the phase $\phi(\omega)$ to all orders. ${ }^{6}$ In principle, this can be accomplished using programmable phase elements such as liquid crystal spatial light modulators. ${ }^{18,19}$ As a result the chirp along with all other higher-order terms is removed and the pulse is optimally compressed. Figure 5(a) depicts the compressed pulse resulting from the central waveguide of the AlGaAs array under such ideal conditions. This latter figure corresponds to the intensity and spectrum shown in Figs. 3(a) and 4(a). In this case the compressed pulse has a 37 fs intensity FWHM duration. On the other hand, we found that under the same conditions [corresponding to Figs. 3 (b) and 4(b)] the best compression possible from a single waveguide will be $54 \mathrm{fs}$ (FWHM), as shown in Fig. 5(b). Figure 5(b) also indicates that in the latter case a significant part of the intensity is contained in a not needed pedestal. Therefore our results indicate that array compressors may be promising in terms of achieving higher compression ratios.

We have also assessed the performance of such array systems when the phase $\phi(\omega)$ is only compensated up to second order $\phi_{2}$ (chirp compensation). This scenario typically arises when either grating or prism pairs are used. In this case we found that for the data corresponding to Figs. 3(a) and 4(a) the best possible compression the array can offer is achieved when $\phi_{2}=0.0216 \mathrm{ps}^{2}$. Under these conditions the compressed pulse has an intensity FWHM of $45 \mathrm{fs}$, as demonstrated in Fig. 5(c). On the other hand, from a single waveguide element [under the same conditions, see
Figs. 3(b) and 4(b)], the compressed pulse shown in Fig. 5(d) has a duration of $55 \mathrm{fs}$ FWHM when $\phi_{2}=0.0258 \mathrm{ps}^{2}$. Again in this case the array compressor performs better.

In conclusion, we have shown that efficient optical pulse compression can be achieved when normally dispersive nonlinear waveguide arrays are used in conjunction with dispersive elements such as gratings or other programmable phase filters. The compression resulting from such discrete arrays was found to be of better quality as compared to that obtained traditionally from a single nonlinear waveguide element. Finally, we note, that even though in this work we have specifically investigated this possibility in AlGaAs arrays, our results can also be applicable in other nonlinear normally dispersive materials.

This work was supported by the Greek Ministry of Education through the Heracletos and Pythagoras grants. R.M. would like to acknowledge support from a NSERC Strategic grant program.

${ }^{1}$ E. B. Treacy, IEEE J. Quantum Electron. QE-5, 454 (1969).

${ }^{2}$ C. V. Shank, R. L. Fork, R. Yen, R. H. Stolen, and W. J. Tomlinson, Appl. Phys. Lett. 40, 761 (1982).

${ }^{3}$ D. Grischkowsky and A. C. Balant, Appl. Phys. Lett. 41, 1 (1982).

${ }^{4}$ W. J. Tomlinson, R. H. Stolen, and C. V. Shank, J. Opt. Soc. Am. B 1, 139 (1984).

${ }^{5}$ R. L. Fork, C. H. Brito Cruz, P. C. Becker, and C. V. Shank, Opt. Lett. 12, 483 (1987).

${ }^{6}$ G. P. Agrawal, Applications of Nonlinear Fiber Optics (Academic, San Diego, 2001).

${ }^{7}$ E. Zeek, K. Maginnis, S. Backus, U. Russek, M. Murnane, G. Mourou, H. Kapteyn, and G. Vdovin, Opt. Lett. 24, 493 (1999).

${ }^{8}$ U. Keller, Nature (London) 424, 831 (2003).

${ }^{9}$ D. E. Spence, P. N. Kean, and W. Sibbett, Opt. Lett. 16, 42 (1991).

${ }^{10}$ D. N. Christodoulides and R. I. Joseph, Opt. Lett. 13, 794 (1988).

${ }^{11}$ D. N. Christodoulides, F. Lederer, and Y. Silberberg, Nature (London) 424, 817 (2003).

${ }^{12}$ H. S. Eisenberg, Y. Silberberg, R. Morandotti, and J. S. Aitchison, Phys. Rev. Lett. 85, 1863 (2000).

${ }^{13}$ R. Morandotti, U. Peschel, J. S. Aitchison, H. S. Eisenberg, and Y. Silberberg, Phys. Rev. Lett. 83, 2726 (1999).

${ }^{14}$ J. W. Fleischer, M. Segev, N. K. Efremidis, and D. N. Christodoulides, Nature (London) 422, 147 (2003).

${ }^{15}$ J. Meier, J. Hudock, D. N. Christodoulides, G. Stegeman, Y. Silberberg, R. Morandotti, and J. S. Aitchison, Phys. Rev. Lett. 91, 143907 (2003).

${ }^{16}$ D. Modotto, J. P. Mondia, S. Linden, H. W. Tan, R. Morandotti, T. C. Kleckner, A. Locatelli, C. De Angelis, H. M. van Driel, C. R. Stanley, and J. S. Aitchison, Opt. Commun. 249, 201 (2005).

${ }^{17}$ S. Droulias, K. Hizanidis, J. Meier, and D. N. Christodoulides, Opt. Express 13, 1827 (2005).

${ }^{18}$ A. Efimov, C. Schaffer, and D. H. Reitze, J. Opt. Soc. Am. B 12, 1968 (1995).

${ }^{19}$ A. M. Weiner, Rev. Sci. Instrum. 71, 1929 (2000). 